\title{
Dose Reduction in Standard Head CT: First Results from a New Scanner Using Iterative Reconstruction and a New Detector Type in Comparison with Two Previous Generations of Multi-slice CT
}

\author{
C. Ozdoba · J. Slotboom · G. Schroth $\cdot$ S. Ulzheimer • \\ R. Kottke $\cdot$ H. Watzal $\cdot$ C. Weisstanner
}

Received: 31 July 2013 / Accepted: 14 October 2013 / Published online: 31 January 2014

(C) The Author(s) 2013. This article is published with open access at Springerlink.com

\begin{abstract}
Purpose Computed tomography (CT) accounts for more than half of the total radiation exposure from medical procedures, which makes dose reduction in CT an effective means of reducing radiation exposure. We analysed the dose reduction that can be achieved with a new CT scanner [Somatom Edge (E)] that incorporates new developments in hardware (detector) and software (iterative reconstruction). Methods We compared weighted volume CT dose index $\left(\mathrm{CTDI}_{\mathrm{vol}}\right)$ and dose length product (DLP) values of 25 consecutive patients studied with non-enhanced standard brain $\mathrm{CT}$ with the new scanner and with two previous models each, a 64-slice 64-row multi-detector CT (MDCT) scanner with 64 rows (S64) and a 16-slice 16-row MDCT scanner with 16 rows (S16). We analysed signal-to-noise and contrast-to-noise ratios in images from the three scanners and performed a quality rating by three neuroradiologists to analyse whether dose reduction techniques still yield sufficient diagnostic quality.

Results $\mathrm{CTDI}_{\mathrm{Vol}}$ of scanner E was 41.5 and $36.4 \%$ less than the values of scanners S16 and S64, respectively; the DLP values were 40 and $38.3 \%$ less. All differences were statistically significant $(p<0.0001)$.
\end{abstract}

C. Weisstanner, MD $(\bowtie) \cdot$ C. Ozdoba, PD $\cdot$ J. Slotboom, PhD · Prof. G. Schroth · R. Kottke, MD · H. Watzal, MD

University Institute of Diagnostic and Interventional Neuroradiology, University of Bern/Inselspital, Freiburgstrasse 4, 3010 Bern, Switzerland

e-mail: christian.weisstanner@insel.ch

S. Ulzheimer, PhD

Computed Tomography, Siemens Healthcare,

Forchheim, Germany
Signal-to-noise and contrast-to-noise ratios were best in S64; these differences also reached statistical significance. Image analysis, however, showed "non-inferiority" of scanner E regarding image quality.

Conclusions The first experience with the new scanner shows that new dose reduction techniques allow for up to $40 \%$ dose reduction while still maintaining image quality at a diagnostically usable level.

Keywords Tomography/X-ray computed/scanners · Dosage/radiation - Sv radiation dose equivalent · Image reconstruction $\cdot$ Neuroimaging
Abbreviations
CNR Contrast-to-noise ratio
CTDI $_{\text {vol }}$ Weighted volume computed tomography dose index
DLP Dose length product
E CT scanner Somatom Edge
MDCT Multi-detector computed tomography
PACS Picture Archiving and Communication System
S16, S64 16-row and 64-row MDCT scanners with 16 and 64 rows, respectively
SNR Signal-to-noise ratio

\section{Introduction}

According to the annual reports published by the German Federal Office for Radiation Protection, computed tomography (CT) accounted for $6.1 \%$ of all radiologic procedures, but $51.9 \%$ of the collective effective dose in 2003; by 2008, these percentages had risen to 8 and $60 \%$, respectively $[1,2]$. As the number of installed CT scanners in industria- 
Fig. 1 Schematic depiction of the principle of Sinogram Affirmed Iterative Reconstruction, the iterative reconstruction approach implemented on the Somatom Definition Edge. Image quality is improved by iterative loops both in image space and raw data space to reduce image noise and artefacts, respectively

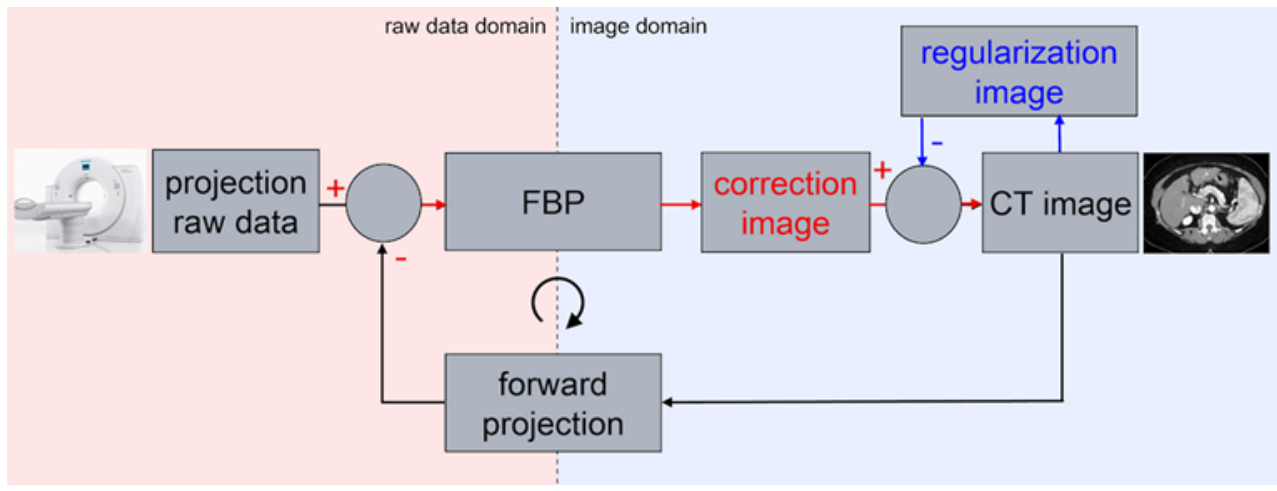

lised countries has steadily increased in the past 2 decades [3], a further rise in both percentages may be expected.

The ever-increasing number of CT studies makes dose reduction one of the most important tasks for manufacturers and operators of CT scanners. This issue can be addressed by developments both in the scanner hardware and software, especially with advanced techniques for image reconstruction that allow to acquire images with reduced dose levels without loss in image quality.

At the authors' institution, the first machine from series production of a new CT scanner (Somatom Definition Edge; Siemens Healthcare, Erlangen, Germany) was installed in March 2012. This system is equipped with a new type of detector and routinely uses iterative reconstruction for image calculation. The detector has a highly integrated design, thereby reducing noise within the detector's circuits, which allows for improved signal yield and reduced dose. Iterative reconstruction allows further reducing the applied dose compared with conventional filtered back projection. The fundamentals of iterative reconstruction have long been known and applied in other fields such as nuclear medicine [4]. Since 2009, CT manufacturers started introducing iterative reconstruction algorithms commercially on their clinical scanners. On the Somatom Definition Edge, an iterative reconstruction approach called Sinogram Affirmed Iterative Reconstruction is implemented.

In an iterative loop, noise is removed from the image by modelling image noise and subtracting the resulting regularisation image from the original data (Fig. 1). In contrast to simple image filters that can also reduce noise but lead to image blurring, iterative reconstruction maintains the high contrast resolution of the image and can remove artefacts from the image. A CT scan is simulated on the existing image data as if a real CT scan would be performed on the reconstructed image data in a step called 'forward-projection'. The result is simulated projection raw data that can then be compared with the actually measured data from the scanner. The differences between the simulated and measured projection raw data can be used to reconstruct an improved image; this can be repeated multiple times until the desired image quality level is reached.

The manufacturer claims that more than $30 \%$ dose reduction is possible depending on the application and the clinical task. In this study, we tested this statement by comparing the dose levels that we achieved in routine imaging with those from the systems previously used for non-enhanced standard brain CT. In addition, we analysed signal-to-noise ratio (SNR) and contrast-to-noise ratio (CNR) to assess whether image quality can be maintained with markedly reduced dose.

\section{Material and Methods}

We compared CT studies in routine head imaging in the first 25 patients studied with the new system with the values from the last 25 patients examined in the Edge's predecessor (Somatom Sensation 64, Siemens Healthcare) and the respective values from a 16-slice multi-detector computed tomography (MDCT) system used in our institution's emergency room (Somatom Sensation 16, Siemens Healthcare). This unselected cohort with numerous different diagnoses and a random age- and sex-mix was chosen to reflect a 'real life' situation.

We used only non-enhanced CT studies of the head in consecutive patients. Weighted volume computed tomography dose index $\left(\mathrm{CTDI}_{\mathrm{vol}}\right)$ and dose length product (DLP) values were taken from the protocols that the scanners automatically send to the hospital's Picture Archiving and Communication System (PACS). The standard examination protocols used in the three scanners are shown in Table 1. CT scanner Somatom Edge (E) was operated in helical mode, whereas incremental acquisition was used in 16-row MDCT scanner with 16 rows (S16) and 64-row MDCT scanner with 64 rows (S64), as these older machines do not allow helical acquisition with a tilted gantry.

As we measured attenuation values (Hounsfield units) in the frontal cortex and white matter, we excluded cases that might present Hounsfield values altered due to parenchyma 
Table 1 Technical parameters of the examination protocols that were used on the three scanners for standard brain studies

\begin{tabular}{|c|c|c|c|}
\hline & $\begin{array}{l}\text { Siemens Soma- } \\
\text { tom Sensation } \\
16(\mathrm{~S} 16)\end{array}$ & $\begin{array}{l}\text { Siemens Soma- } \\
\text { tom Sensation } \\
64(\mathrm{~S} 64)\end{array}$ & $\begin{array}{l}\text { Siemens Soma- } \\
\text { tom Definition } \\
\text { Edge (E) }\end{array}$ \\
\hline Gantry angulation & Cantho-meatal & Cantho-meatal & Cantho-meatal \\
\hline $\mathrm{mAs}$ & 220 & 380 & 230 \\
\hline $\begin{array}{l}\text { Kiloelectron volt } \\
(\mathrm{keV})\end{array}$ & 120 & 120 & 100 \\
\hline $\begin{array}{l}\text { Field of view } \\
(\mathrm{mm})\end{array}$ & 220 & 220 & 220 \\
\hline $\begin{array}{l}\text { Slice thickness/ } \\
\text { table feed (mm) }\end{array}$ & $4.5 / 4.5$ & $4.8 / 4.8$ & $3.0 / 3.0$ \\
\hline $\begin{array}{l}\text { Reconstruction } \\
\text { kernel }\end{array}$ & $\mathrm{H} 41 \mathrm{~s}$ & $\mathrm{H} 41 \mathrm{~s}$ & $\mathrm{~J} 45 \mathrm{~s}$ \\
\hline
\end{tabular}

compression or oedema, i.e. patients with space-occupying lesions, hydrocephalus and intracranial or intracerebral haemorrhage.

The study was approved by the institutional review board. All statistical analyses were performed with GraphPad Prism version 6.01 (GraphPad Software, La Jolla, CA).

Weighted Volume Computed Tomography Dose Index and Dose Length Product Values

In the first step, values were tested for normal (Gaussian) distribution with the D'Agostino-Pearson omnibus normality test. Unpaired $t$-tests with a confidence interval of $95 \%$ were performed for each possible pair of the three scanners to test for significant differences.

\section{Signal-to-Noise and Contrast-to-Noise Ratios}

The 'statistics/circle' function of our hospital's PACS (Sectra Imtec AB, Stockholm, Sweden; IDS 5, Release 11.4.1) was used to determine Hounsfield values in regions of interest in the frontal cortex and adjacent white matter. The same-size region of interest was used in all patients; measured mean values and standard deviation (SD) were used for subsequent analysis. A typical example of the regions used is shown in Fig. 2.

The mean/SD quotient was calculated to determine the SNR. CNR was calculated according to the formula by Mullins et al. [5].

$$
\frac{(\text { mean } \mathrm{GM}-\text { mean WM })}{\left((\mathrm{SD} \cdot \mathrm{GM})^{2}+(\mathrm{SD} \cdot \mathrm{WM})^{2}\right)^{0.5}}
$$

where

GM Grey matter

WM White matter

SD Standard deviation

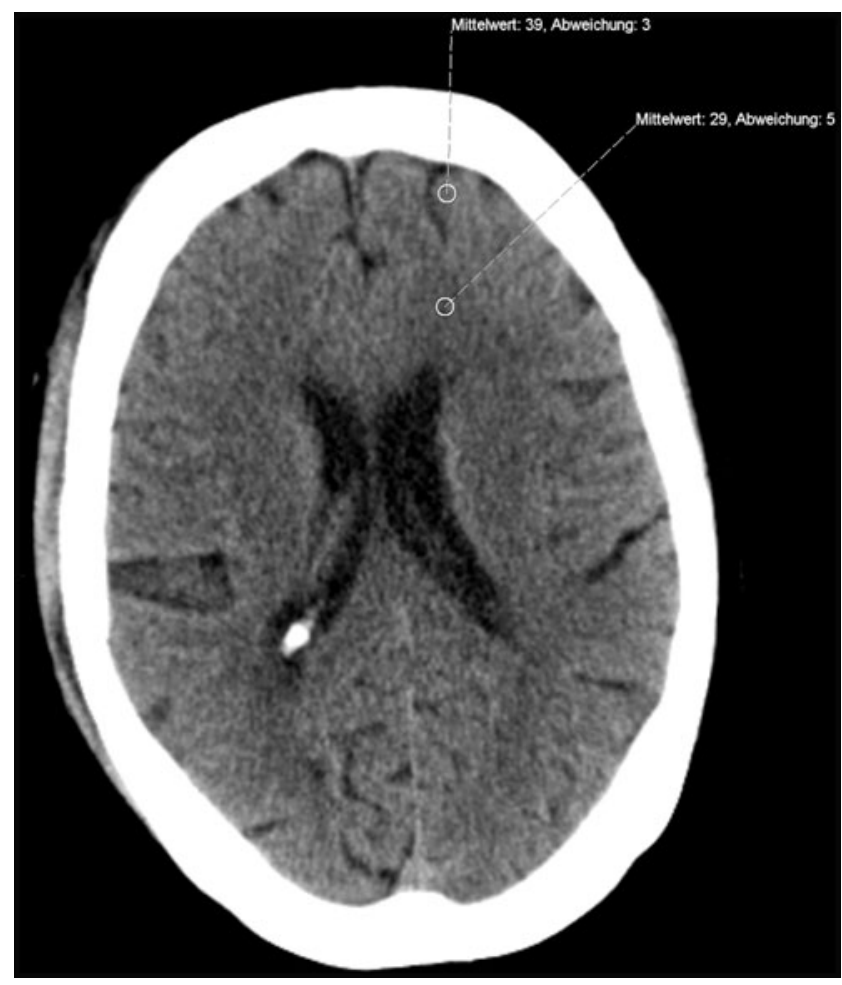

Fig. 2 Typical region-of-interest measurements in the frontal cortex and white matter (diameter of region of interest: $5 \mathrm{~mm}$ ). Mittelwert mean, Abweichung standard deviation

The non-parametric Mann-Whitney test (two-tailed, 95\% confidence interval) was used to test for significant differences between these values, as these data did not show Gaussian distribution.

\section{Image Quality Analysis}

Three board-certified radiologists with a neuroradiological experience of at least 2 years assessed image quality in all $75 \mathrm{CT}$ studies. The raters were blinded to the type of scanner used; studies were presented in a random manner. Image quality was evaluated in the supratentorial parenchyma, the basal ganglia (especially with delineation of the insular ribbon sign) and the infratentorial parenchyma. Image quality readings were performed on PACS workstations with reporting-standard monitors; the raters were free to subjectively adjust window/level settings. Readers were free not to evaluate images that were degraded due to artefacts. Image quality was graded from 1 to 5 :

1. not diagnostic, unusable,

2. reduced quality, diagnostic unsure, better repeat examination,

3. medium quality, still diagnostic,

4. slightly reduced, allows safe diagnostic,

5. good quality, diagnostically perfectly usable. 
Table 2 Demographic characteristics of the patients studied in the three scanners

\begin{tabular}{llll}
\hline & $\begin{array}{l}\text { Siemens Soma- Siemens Soma- } \\
\text { tom Sensation } \\
16(\mathrm{~S} 16)\end{array}$ & $\begin{array}{l}\text { Siemens Soma- } \\
\text { tom Sensation } \\
\text { tom Definition } \\
\text { Edge (E) }\end{array}$ \\
\hline Sex (male/female) & $13 / 12$ & $16 / 9$ & $14 / 11$ \\
Age, mean (years) & 49.6 & 59.0 & 60.3 \\
$\begin{array}{l}\text { Age, median } \\
\text { (years) }\end{array}$ & 48.2 & 60.8 & 64.8 \\
$\begin{array}{l}\text { Age range } \\
\text { (min-max; years) }\end{array}$ & $17.3-89.5$ & $19.5-90.3$ & $20.7-90.0$ \\
\hline
\end{tabular}

Table 3 CTDI $_{\mathrm{vol}}$ and DLP values measured in the three scanners. Statistical analysis shows a marked dose reduction in scanner E compared with the other models

\begin{tabular}{|c|c|c|c|}
\hline & $\begin{array}{l}\text { Siemens Soma- } \\
\text { tom Sensation } \\
16(\mathrm{~S} 16)\end{array}$ & $\begin{array}{l}\text { Siemens Soma- } \\
\text { tom Sensation } \\
64(\mathrm{~S} 64)\end{array}$ & $\begin{array}{l}\text { Siemens Soma- } \\
\text { tom Definition } \\
\text { Edge (E) }\end{array}$ \\
\hline \multicolumn{4}{|l|}{$\overline{C T D I(m G y)}$} \\
\hline Mean & 59.63 & 54.86 & 34.88 \\
\hline Median & 58.49 & 54.94 & 34.72 \\
\hline $\begin{array}{l}\text { Standard } \\
\text { deviation (SD) }\end{array}$ & 2.80 & 2.93 & 4.79 \\
\hline \multicolumn{4}{|l|}{$D L P(m G y c m)$} \\
\hline Mean & 975.16 & 952.82 & 574.12 \\
\hline Median & 933.00 & 906.49 & 559.00 \\
\hline SD & 82.62 & 79.78 & 82.33 \\
\hline $\begin{array}{l}\text { Statistical } \\
\text { comparison }\end{array}$ & $t$-test CTDI & Difference $\%$ & \\
\hline S16/S64 & $p<0.0001$ & 8.00 & \\
\hline $\mathrm{S} 16 / \mathrm{E}$ & $p<0.0001$ & 41.51 & \\
\hline \multirow[t]{2}{*}{ S64/E } & $p<0.0001$ & 36.42 & \\
\hline & $t$-test DLP & & \\
\hline S16/S64 & $p=0.3357$ & 2.84 & \\
\hline $\mathrm{S} 16 / \mathrm{E}$ & $p<0.0001$ & 40.06 & \\
\hline S64/E & $p<0.0001$ & 38.33 & \\
\hline
\end{tabular}

The ratings were statistically compared in the same way as the CTDI ${ }_{\text {vol }}$ and DLP values.

\section{Results}

Demographic data of the patients are given in Table 2.

Weighted Volume Computed Tomography Dose Index and Dose Length Product Values

The D'Agostino-Pearson test showed normal distribution for five of the six values; the only exception was CTDI in S16. We still performed $t$-tests on all values taking into account that the results involving CTDI ${ }_{\mathrm{vol}}$ in S16 may be imprecise. The CTDI ${ }_{\mathrm{vol}}$ and DLP values and the results of the statistical comparison are listed in Table 3 and shown in Figs. 3 and 4.

\section{$\mathrm{CTDI}_{\mathrm{Vol}}$ values in the three scanners \\ (mean \pm standard deviation)}

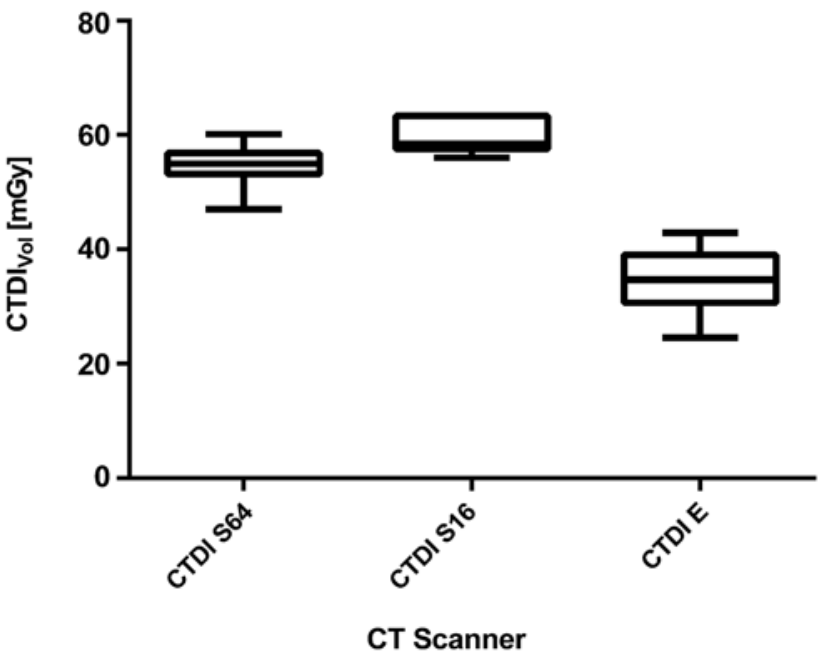

Fig. 3 Weighted volume computed tomography dose index $\left(\mathrm{CTDI}_{\mathrm{vol}}\right)$ values in the three scanners. The box indicates the range (minimummaximum), and the horizontal bar in the box denotes the mean value. Scanner 'CT scanner Somatom Edge's' (E's) values are markedly reduced in comparison with the two older scanners

\section{DLP values in the three scanners (mean \pm standard deviation)}

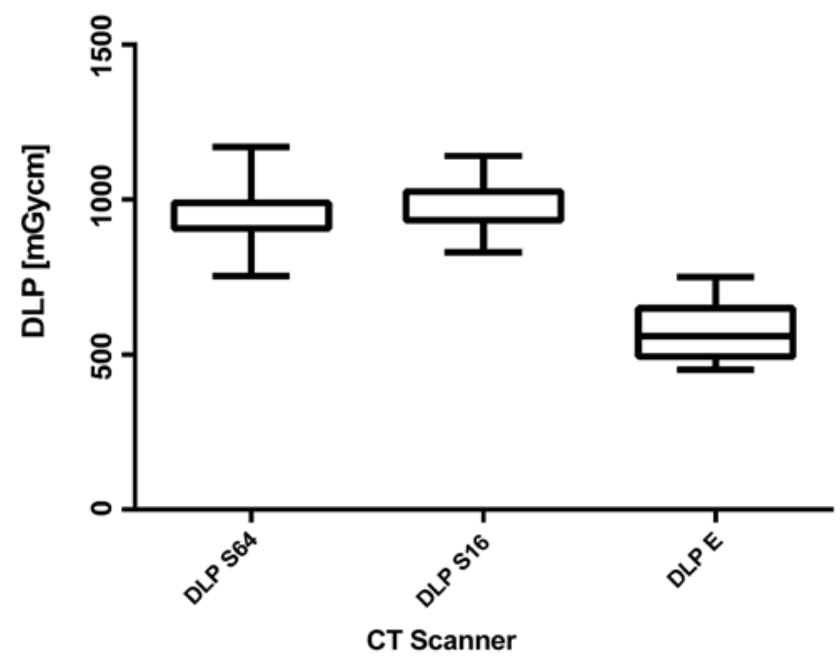

Fig. 4 Dose length product (DLP) values in the three scanners. The box indicates the range (minimum-maximum), and the horizontal bar in the box denotes the mean value. The 16-row multi-detector computed tomography (MDCT) scanners with 16 rows (S16) and 64-row MDCT scanners with 64 rows (S64) show no visible difference, whereas the values for CT scanner Somatom Edge are markedly lower

S64 had statistically significantly lower CTDI ${ }_{\text {vol }}$ than S16 $(p<0.0001)$; the absolute difference was $8 \%$. E was between 36 and $41 \%$ lower in both parameters than the two older scanners; these differences were highly significant as well ( $p<0.0001$ in both instances).

Statistical comparison of the DLP values showed no significant difference between S16 and S64, whereas the 
Table 4 Signal-to-noise ratio in cortex and white matter and contrastto-noise ratios in the three scanners. S64 shows significantly better values than S16 and E (discussion)

\begin{tabular}{|c|c|c|c|}
\hline & $\begin{array}{l}\text { Siemens Soma- } \\
\text { tom Sensation } \\
16(\mathrm{~S} 16)\end{array}$ & $\begin{array}{l}\text { Siemens Soma- } \\
\text { tom Sensation } \\
64 \text { (S64) } \\
\end{array}$ & $\begin{array}{l}\text { Siemens Soma- } \\
\text { tom Definition } \\
\text { Edge (E) }\end{array}$ \\
\hline \multicolumn{4}{|l|}{ SNR (cortex) } \\
\hline Mean & 12.12 & 16.19 & 10.12 \\
\hline $\begin{array}{l}\text { Standard devia- } \\
\text { tion (SD) }\end{array}$ & 3.43 & 7.88 & 2.00 \\
\hline \multicolumn{4}{|c|}{ SNR (white matter) } \\
\hline Mean & 8.70 & 12.69 & 7.41 \\
\hline $\mathrm{SD}$ & 1.97 & 5.55 & 1.84 \\
\hline \multicolumn{4}{|c|}{ Contrast-to-noise (cortex/white matter) } \\
\hline Mean & 1.59 & 3.71 & 2.19 \\
\hline SD & 0.42 & 1.52 & 0.61 \\
\hline $\begin{array}{l}\text { Statistical } \\
\text { comparison }\end{array}$ & $p$-value & & \\
\hline \multicolumn{4}{|l|}{ SNR $($ cortex $)$} \\
\hline S16/S64 & 0.0230 & & \\
\hline $\mathrm{S} 16 / \mathrm{E}$ & 0.0434 & & \\
\hline S64/E & $<0.0001$ & & \\
\hline \multicolumn{4}{|c|}{ SNR (white matter) } \\
\hline S16/ S64 & $<0.0001$ & & \\
\hline $\mathrm{S} 16 / \mathrm{E}$ & 0.0213 & & \\
\hline S64/E & $<0.0001$ & & \\
\hline \multicolumn{4}{|l|}{ CNR } \\
\hline S16/S64 & $<0.0001$ & & \\
\hline $\mathrm{S} 16 / \mathrm{E}$ & $<0.0007$ & & \\
\hline $\mathrm{S} 64 / \mathrm{E}$ & $<0.0001$ & & \\
\hline
\end{tabular}

$C N R$ contrast to noise ratio, $S N R$ signal to noise ratio

values obtained from $\mathrm{E}$ were significantly lower than those from both S16 and S64 ( $p<0.0001$ in both instances).

\section{Signal-to-Noise and Contrast-to-Noise Ratios}

The majority of the values did not pass the test for normal distribution. Therefore, data were compared with the nonparametric Mann-Whitney test. The results are listed in Table 4.

The SNR and CNR of the Somatom Edge are lower than those measured in the two older scanners; these values reached statistical significance $(p<0.0001)$ only in comparison with the 64-row scanner. The S64 performed best in this comparison (SNR in white matter was 12.69 compared with 8.7 for the S16 and 7.41 for the E; CNR was 3.71 compared with 1.59 for the S16 and 2.19 for the E).

Image Quality Evaluation

The mean ratings are listed in Table 5, and the statistical comparisons are found in Table 6. An intra-rater analysis for the different scanners and anatomical regions showed no statistically significant differences between the three
Table 5 Subjective image quality evaluation by the three raters. Although the dose in E was significantly lower than in S16 and S64, the readings mostly did not reveal significant differences (exception and details in the text)

\begin{tabular}{llll}
\hline & Rater 1 & Rater 2 & Rater 3 \\
\hline S16 & & & \\
Supratentorial & 4.68 & 3.67 & 3.44 \\
Basal ganglia & 4.40 & 4.04 & 3.16 \\
Infratentorial & 3.76 & 3.61 & 3.24 \\
S64 & & & \\
Supratentorial & 3.80 & 3.78 & 3.72 \\
Basal ganglia & 3.92 & 3.74 & 3.68 \\
Infratentorial & 3.56 & 3.78 & 3.68 \\
E & & & \\
Supratentorial & 4.08 & 4.44 & 4.36 \\
Basal ganglia & 3.92 & 4.52 & 4.08 \\
Infratentorial & 3.72 & 3.92 & 4.16 \\
\hline
\end{tabular}

Table 6 Inter-rater variability of subjective image quality ratings

\begin{tabular}{llll}
\hline & $\begin{array}{l}\text { Rater 1 } \\
(p \text {-values })\end{array}$ & $\begin{array}{l}\text { Rater 2 } \\
(p \text {-values })\end{array}$ & $\begin{array}{l}\text { Rater 3 } \\
(p \text {-values })\end{array}$ \\
\hline Supratentorial S16/S64 & $<0.0001$ & 0.7610 & 0.2321 \\
Supratentorial S16/E & 0.0004 & 0.0012 & $<0.0001$ \\
Supratentorial S64/E & 0.1056 & 0.0096 & 0.0048 \\
Basal ganglia S16/S64 & 0.0216 & 0.2435 & 0.0137 \\
Basal ganglia S16/E & 0.0305 & 0.0369 & $<0.0001$ \\
Basal ganglia S64/E & $>0.9999$ & 0.0007 & 0.0961 \\
Infratentorial S16/S64 & 0.4877 & 0.5694 & 0.0808 \\
Infratentorial S16/E & 0.8052 & 0.3757 & 0.0002 \\
Infratentorial S64/E & 0.5754 & 0.8374 & 0.0574 \\
\hline
\end{tabular}

Statistically significant differences $(p<0.0001)$ are given in italics

machines with two exceptions: the most experienced rater's (more than 25 years in neuroradiology) evaluation yielded a statistically significant $(p<0.0001)$ advantage in scanner E in comparison with S16 for supratentorial parenchyma and basal ganglia. In some instances (Tables 5 and 6), statistical difference, with advantages for the older models, was missed very closely, but scanner E never lost a direct comparison significantly.

\section{Discussion}

The use of a new detector technology and the exclusive use of iterative reconstruction in the standard head clinical scanning protocol on the new scanner resulted in a marked reduction of radiation exposure to the patient; the difference of approximately $40 \%$ that was established in clinical routine is higher than previous manufacturer's estimates.

CTDI $_{\text {vol }}$ and DLP are suitable parameters for such a study, as they are objective technical parameters for the applied dose. In a recent overview, Huda and Mettler [6] came to the conclusion that these values can be used clinically, as they can be easily obtained from the scanner's patient protocol; 
DLP allows estimating the patient's effective dose. For the purpose of this study, these parameters therefore provide a good estimate and allow comparing dose levels from various scanners.

The increasing awareness for dose reduction has led to the introduction of 'Diagnostic Reference Values' by numerous organisations, which have partly been implemented in local legislation. The Swiss Federal Office of Public Health has defined this value for a standard brain CT study as a $\mathrm{CTDI}_{\text {vol }}$ of $65 \mathrm{mGy}$ and a DLP of 1,000 mGycm. The 'target values' were set at $45 \mathrm{mGy}$ and $600 \mathrm{mGycm}$, respectively [7]. In the United States, the applied dose has been markedly reduced since the introduction of the American College of Radiology's (ACR) CT accreditation program [8]; currently, the ACR's reference value for a head CT is a CTDI $_{\text {vol }}$ of 75 mGy [9].

With a CTDI ${ }_{\text {vol }}$ of less than $35 \mathrm{mGy}$ for an adult head CT, the new scanner easily underruns these recommendations.

Although responsible CT operators will welcome the new technology that allows for such marked decrease of patient dose, they will not accept a system where these reduced doses mean a reduction in image quality. Our results show that, although the dose is markedly reduced, the new scanner's image quality fulfils the criteria of what is called 'non-inferiority' in clinical drug studies with regard to diagnostic usability despite reduced SNR and CNR. Although the subjective readers' evaluations differed in some aspects, the older machines were never rated better than the new machine with statistical significance. We used a subjective readers' rating instead of phantom studies for the same reason why we studied an unselected patient population: we wanted a 'real-life' test scenario that seemed more appropriate to evaluate a new system in practical use (when evaluating SNR, it should be noted that the protocol on the new scanner uses $3-\mathrm{mm}$ sections compared with $4.5 / 4.8 \mathrm{~mm}$ in the older models; this change was introduced to improve diagnostic accuracy in routine imaging in cases of, e.g. trauma, subarachnoid haemorrhage or suspected cerebral ischaemia). A similar study with only one reader and a $30 \%$ dose reduction has shown that image quality was not compromised [10]; in this study, a CTDI ${ }_{\mathrm{Vol}}$ of $42.3 \mathrm{mGy}$ and a mean DLP of $733 \mathrm{mGycm}$ were achieved. Our data suggest that keeping up image quality is possible with even further reduction.

In conclusion, we may fairly assume from recent developments that the trend to an ever-increasing number of CT installations and CT studies will probably continue. These first data show that the use of state-of-the-art technology provides a way to keep up diagnostic quality while reducing the general population's radiation exposure.
Further studies will have to be conducted to show whether more refined image reconstruction protocols will be able to improve image quality more markedly than just 'non-inferior'; first results are promising [11]. For the time being, it can be stated that iterative reconstruction in combination with a high-yield detector is a very promising achievement in current CT technology with regard to dose reduction.

Conflict of Interest The authors declare that there are no actual or potential conflicts of interest in relation to this article.

Open Access This article is distributed under the terms of the Creative Commons Attribution Noncommercial License which permits any noncommercial use, distribution, and reproduction in any medium, provided the original author(s) and the source are credited.

\section{References}

1. Umweltradioaktivität und Strahlenbelastung. Jahresbericht 2005. Bundesministerium für Umwelt, Naturschutz und Reaktorsicherheit (ed.). http://nbn-resolving.de/urn:nbn:de:0221-20100331990. Accessed 26 July 2013.

2. Umweltradioaktivität und Strahlenbelastung. Jahresbericht 2009. Bundesministerium für Umwelt, Naturschutz und Reaktorsicherheit (ed.). http://nbn-resolving.de/urn:nbn:de:0221-201103025410. Accessed 26 July 2013.

3. OECD. Health at a Glance 2011: OECD Indicators, OECD Publishing. (2011) http://dx.doi.org/10.1787/health_glance-2011-en (2011). Accessed 26 July 2013.

4. Li J, Jaszczak RJ, Greer KL, Coleman RE, et al. Implementation of an accelerated iterative algorithm for cone-beam SPECT. Phys Med Biol. 1994;39:643-53.

5. Mullins ME, Lev MH, Bove P, et al. Comparison of Image Quality Between Conventional and Low-Dose Nonenhanced Head CT. AJNR Am J Neuroradiol. 2004;25:533-8.

6. Huda W, Mettler FA. Volume CT dose index and dose-length product displayed during CT: what good are they? Radiology. 2011;258:236-42.

7. Bundesamt für Gesundheit, Abteilung Strahlenschutz. Merkblatt R-06-06: Diagnostische Referenzwerte in der Computertomographie. Eidgenössisches Departement des Innern EDI, April 1, 2010. http://www.bag.admin.ch/themen/strahlung/10463/10958/index. html?lang=de. Accessed 26 July 2013.

8. McCollough C, Branham T, Herlihy V, et al. Diagnostic reference levels from the ACR CT accreditation program. J Am Coll Radiol. 2011;8:795-803.

9. ACR Practice Guideline for Diagnostic Reference Levels in Medical X-Ray Imaging. Revised 2008 (Resolution 3). Available at: http://www.acr.org/ /media/ACR/Documents/PGTS/guidelines/ Reference_Levels.pdf. Accessed 26 July 2013.

10. Korn A, Fenchel M, Bender B, et al. Iterative reconstruction in head CT: image quality of routine and low-dose protocols in comparison with standard filtered back-projection. AJNR Am J Neuroradiol. 2012;33:218-24.

11. Buhk JH, Laqmani A, von Schultzendorff HC, et al. Intraindividual evaluation of the influence of iterative reconstruction and filter kernel on subjective and objective image quality in computed tomography of the brain. Fortschr Röntgenstr. 2013;185:741-8. 